\title{
Prevalence of Lumbosacral Transitional Vertebra in Individuals with Low Back Pain: Evaluation Using Plain Radiography and Magnetic Resonance Imaging
}

\author{
Asra Shaikh ${ }^{1}$, Sohail Ahmed Khan ${ }^{1}$, Munawar Hussain ${ }^{1}$, Sadia Soomro ${ }^{1}$, Hatem Adel ${ }^{1}$, \\ Syed Omair Adil $^{2}$, Farheen Huda ${ }^{1}$, Usman Khanzada ${ }^{1}$ \\ ${ }^{1}$ Dow Institute of Radiology, Dow University of Health Sciences, Karachi, Pakistan \\ ${ }^{2}$ Department of Research, Dow University of Health Sciences, Karachi, Pakistan
}

\begin{abstract}
Study Design: Descriptive cross-sectional study.
Purpose: To determine the frequency of lumbosacral transitional vertebrae (LSTV) in patients with low back pain (LBP) and the role of iliolumbar ligament (ILL) origin from L5 in LSTV cases.

Overview of Literature: Transitional vertebrae are developmental variants of the spine. LSTV is a common congenital abnormality, and failure to recognize this anomaly may result in serious consequences during surgery.

Methods: All patients aged 11-90 years of either gender with LBP for any duration, who presented for X-ray and magnetic resonance imaging (MRI) of the lumbosacral spine, were included. $X$-rays of the lumbosacral spine in anteroposterior and lateral views were acquired. In addition, T1- and T2-weighted sagittal and axial MRI was performed. Images were evaluated on a workstation.

Results: Of 504 patients, transitional vertebrae were observed in 75 patients (15\%). Among them, $39(52 \%)$ patients had Castellvi type III and 36 (48\%) patients had Castellvi type II. However, on MRI, 42 (56\%) patients had O'Driscoll type II, 18 (24\%) patients had O'Driscoll type IV, and 15 patients (20\%) had O'Driscoll type III. ILL origin from L5 was significantly higher ( $\mathrm{n}=429,100 \%)$ among patients with a normal lumbosacral junction than among patients with a transitional lumbosacral junction ( $n=22,29.3 \%)(p<0.001)$.

Conclusions: LSTV occurs at a high frequency in patients with LBP. Furthermore, in the presence of LSTV, the ILL is not a reliable marker for the identification of $\mathrm{L} 5$.
\end{abstract}

Keywords: Low back pain; Magnetic resonance imaging; Lumbar vertebrae

\section{Introduction}

Lumbosacral transitional vertebra (LSTV) is a common congenital abnormality of the lumbosacral spine. It is described as a condition in which there is complete or partial fusion of the enlarged transverse process of the lowest lumbar vertebral body with the sacrum, termed as sacral- ized or partially sacralized L5. The S1 vertebra can also be seen as separate from the sacrum termed as lumbarized S1. This condition was first described by Bertolotti [1] and is sometimes referred to as Bertolotti syndrome. The prevalence of this common condition has ranged from $4 \%$ to $35.6 \%$ in the literature from various populations [2-4].

Pain in the lower back is common [5] and is a frequent

Received Feb 8, 2017; Revised Mar 20, 2017; Accepted Apr 12, 2017

Corresponding author: Hatem Adel

Dow University of Health Sciences, Dow Institute of Radiology, Gulzar-e-Hijri, Ojha Campus, Suparco Road, KDA Scheme-33, Karachi 75330, Pakistan

Tel: +92-333-3568014, Fax: +92-21-99215763, E-mail: hatemadil89@gmail.com 
Table 1. Magnetic resonance imaging protocol of lumbosacral spine

\begin{tabular}{lccc} 
Imaging parameter & Sagittal T1 & Sagittal T2 & Axial T2 \\
Time to repeat & $400-600$ & $3,000-4,000$ & $3,000-4,000$ \\
Time to echo & $15-25$ & $80-120$ & $80-120$ \\
Slice thickness $(\mathrm{mm})$ & $3-4$ & $3-4$ & $3-4$ \\
Field of view $(\mathrm{mm})$ & 320 & 320 & 200 \\
\hline Matrix & $320 \times 224$ & $320 \times 224$ & $320 \times 192$ \\
\hline
\end{tabular}

cause of morbidity in old and young people alike. The lifetime prevalence of this condition ranges from $60 \%$ to $85 \%$ [6-8], and it can cause a significant loss of work hours, particularly in young patients. Magnetic resonance imaging (MRI) is the initial imaging modality of choice in cases of low back pain (LBP). With the frequent use of MRI, the role of radiography in the evaluation of LBP is now limited. It is important to identify LSTV on MRI to prevent complications during surgical management that may arise because of variant anatomy. Although LBP is frequently related to degenerative disc disease, studies have revealed that it is also associated with LSTV. Previous plain radiography-based studies have shown that LSTV types II and IV are strongly correlated with LBP $[3,9]$.

The radiographic classification of LSTV has been well established by Castellvi [10]. Transitional lumbosacral junction morphology is well appreciated on sagittal MRI. Based on MRI findings, O'Driscoll et al. [11] divided the transitional lumbosacral junction into four types according to the presence of disc material in the uppermost sacral segments.

On axial images, a normal L5 can be identified by the origin of the iliolumbar ligament (ILL) in the presence of a normal lumbosacral junction. Different cadaveric and MRI-based studies have shown the origin of ILL from L5 [12-14]. However, scarce data exist on the origin of ILL in the presence of a transitional lumbosacral junction. Therefore, we had three major aims: (1) to determine the prevalence of LSTV in patients with LBP, (2) to determine the association of LSTV with age and gender, and (3) to assess the reliability of the origin of ILL from L5 in the presence of LSTV.

\section{Materials and Methods}

We retrospectively reviewed the findings of MRI and radiography performed from January 1, 2016, to June 30, 2016. All patients aged 11-90 years of either gender who presented with lower back pain for any duration and underwent both MRI and X-ray of the lumbosacral spine were included in this study. Patients with a history of traumatic injury to the spine or vertebral column, infectious diseases involving the spine, and a history of spinal surgery were excluded.

This study is approved by the Research Evaluation Unit (REU) of College of Physicians and Surgeons of Pakistan (RAD-2012-256-1429). The requirement of informed consent was waived as the data were retrieved from Picture Archiving and Communication System (PACS).

\section{MRI procedure and image analysis}

In all patients, MRI of the lumbosacral spine was performed using either a 0.4 -T or 1.5-T scanner (GE Medical Systems, Milwaukee, WI, USA). Both T1-weighted spinecho images in the sagittal plane and T2-weighted fastspin images in the sagittal and axial planes were acquired. Coronal localizer images, if available, were evaluated for the articulation of the transverse processes with the sacrum. Images of the entire lumbosacral spine were acquired as per routine protocol. Image interpretation was performed on a workstation by a radiologist with experience of $>5$ years in reporting MRI scans as a consultant radiologist. Standard imaging protocols used for MRI are summarized in Table 1.

\section{X-ray procedure}

Plain radiographs of the pelvis in anteroposterior and lateral views were acquired. Radiographs were acquired through digital radiography using a CR radiography system (Fuji Medical Systems, USA Inc., Stamford, CT, USA) with exposure settings of $60-90 \mathrm{kV}$ and $30 \mathrm{mAs}$. Image interpretation was performed on workstation by a consultant radiologist with more than 5 years of experience in reporting of plain radiographs. 


\section{Classification of LSTV}

$\mathrm{X}$-rays and MRI scans were evaluated together for the presence (or absence) of a transitional lumbosacral junction. Reviewers were not blinded to each other's results. Initially, plain radiographs were reviewed, followed by MRI scans. Lumbosacral transitional vertebrae on X-rays were classified according to the Castellvi classification (Table 2) [10].

Following the identification of the lumbosacral junction on plain radiographs, LSTV on MRI scans was labeled according to the method of O'Driscoll et al. [11] (Table 3). Axial cuts at the level of the last lumbar vertebra in both normal and transitional vertebrae cases were evaluated for the presence of ILL. ILL appears as a hypointense band arising from the transverse process of the last lumbar vertebra and extending up to the iliac crest. Different studies have shown that ILL arises from L5 $[12,13,15]$.

\section{Data analysis}

Statistical analysis was performed using SPSS software ver.

Table 2. Castellvi classification of LSTV [10]

\begin{tabular}{ll}
$\begin{array}{l}\text { Type } \\
\text { Type I: Dysplastic } \\
\text { transverse process }\end{array}$ & $\begin{array}{l}\text { Unilateral (A) or bilateral (B) dysplastic } \\
\text { transverse process with height }>19 \mathrm{~mm}\end{array}$ \\
$\begin{array}{ll}\text { Type II: Incomplete lum- } \\
\text { barization/sacralization }\end{array}$ & $\begin{array}{l}\text { Enlarged transverse process with unilateral } \\
\text { (A) or bilateral (B) pseudoarthrosis with the } \\
\text { adjacent sacral ala }\end{array}$ \\
\hline $\begin{array}{l}\text { Type III: Complete lum- } \\
\text { barization/sacralization }\end{array}$ & $\begin{array}{l}\text { Enlarged transverse process, which has a } \\
\text { unilateral (A) or bilateral (B) complete fusion } \\
\text { with the adjacent sacral ala }\end{array}$ \\
\hline Type IV: Mixed & $\begin{array}{l}\text { Type II on one side and type III on the other } \\
\text { side }\end{array}$ \\
\hline
\end{tabular}

LSTV, lumbosacral transitional vertebrae.

Table 3. O'Driscoll transitional lumbosacral junction morphology [11]

\begin{tabular}{ll} 
LSTV type & \multicolumn{1}{c}{ Morphology between S1 and S2 } \\
\hline Type I & No disc material present \\
\hline Type II & Small disc present \\
Type III & $\begin{array}{l}\text { Well-developed disc extending up to whole AP diam- } \\
\text { eter of sacrum }\end{array}$ \\
Type IV & $\begin{array}{l}\text { Well-developed disc extending up to complete AP } \\
\text { diameter of sacrum with abnormal outline of upper } \\
\text { sacrum. }\end{array}$
\end{tabular}

LSTV, lumbosacral transitional vertebrae; AP, anteroposterior.
22 (IBM Corp., Armonk, NY, USA). The mean and standard deviation were calculated for the age of the patients. Frequency and percentages were calculated for variables such as age categorization ( $<20$ years, $20-40$ years, and $>40$ years), gender, and the presence of transitional vertebrae on X-ray and MRI. To determine the differences in the X-ray and MRI characteristics according to age, the unpaired $t$ test and one-way analysis of variance were applied; for the age categories and sex, the chi-square and fisher exact test were applied. A $p$-value of $<0.05$ was considered significant.

\section{Results}

Among 504 patients, the mean age was $43.26 \pm 14.32$ years (range, 13-82 years). Majority of the patients $(\mathrm{n}=282$, $56 \%)$ were older than 40 years, followed by those aged $20-40$ years $(\mathrm{n}=195,38.7 \%)$; only 27 patients $(5.4 \%)$ were younger than 20 years. The number of male patients $(n=267,53 \%)$ was slightly higher than the number of female patients $(\mathrm{n}=237,47 \%)$.

$\mathrm{X}$-ray and MRI analysis revealed that majority $(\mathrm{n}=429$, $85.1 \%$ ) of the patients had normal vertebrae, whereas transitional vertebrae were observed in 75 patients (14.9\%). Of these 75 patients, 39 (52\%) had Castellvi type III and 36 (48\%) had Castellvi type II. Based on MRI findings, 42 patients (56\%) had O'Driscoll type II, 18 (24\%) patients had O'Driscoll type IV, and 15 patients (20\%) had O'Driscoll type III.

The frequency of ILL origin from L5 was significantly higher $(n=429,100 \%)$ among patients with a normal lumbosacral junction than among patients with a transitional lumbosacral junction ( $n=22,29.3 \%$; $p<0.001)$ (Fig. 1).

Based on X-ray findings, patient age was significantly

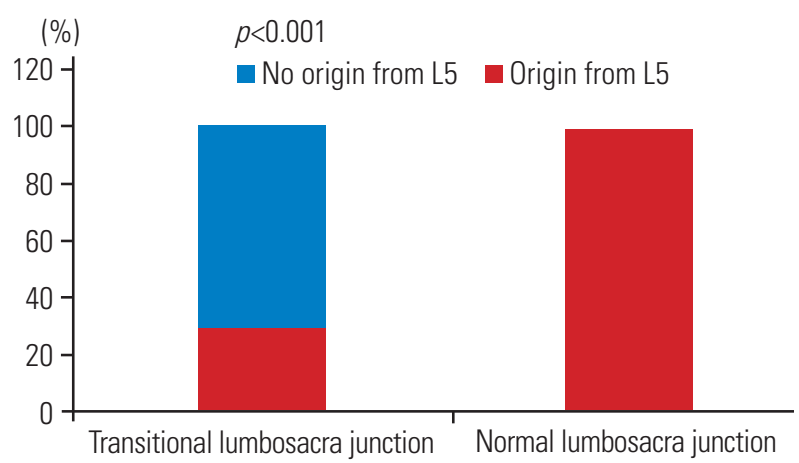

Fig. 1. Comparison of transitional vertebrae with iliolumbar ligament origin from $\mathrm{L} 5$. 
higher among those with Castellvi type III than among those with Castellvi type II ( $48.92 \pm 10.44$ vs. $40.66 \pm 12.64$, $p=0.003$ ). Conversely, patient age was significantly higher among those with O'Driscoll type III than among those with types II and IV (52.8 \pm 6.97 vs. $44.42 \pm 13.92$ and $39.66 \pm 7.48, p=0.007$ ) (Table 4) based on MRI findings.

\section{Discussion}

The results of this study reveal a considerable frequency of LSTV. A comparison of this finding with similar studies in patients with LBP revealed somewhat similar results reported by Nardo et al. [3] and Tang et al. [9]. However, Olofin et al. [16], Delport et al. [17], and Masud et al. [18] have reported a higher prevalence of LSTV and Oyinloye et al. [19] have reported a lower prevalence than that described in this study. This variability may be due to differences in the sample size and age groups. Moreover, the prevalence of LSTV was higher in studies that selected lower back pain patients, whereas lower prevalence was

Table 4. Comparison of LSTV based on castellvi classification and O'Driscoll classification ( $n=75)$

\begin{tabular}{|c|c|c|c|c|c|c|c|}
\hline \multirow{2}{*}{ Variable } & \multicolumn{3}{|c|}{ X-ray } & \multicolumn{4}{|c|}{ MRI } \\
\hline & II $(n=36)$ & III (n=39) & $p$-value & II $(\mathrm{n}=42)$ & III (n=15) & IV (n=18) & $p$-value \\
\hline Age group (yr) & $40.66 \pm 12.64$ & $48.92 \pm 10.44$ & $0.003^{\mathrm{a}), *}$ & $44.42 \pm 13.92$ & $52.8 \pm 6.97$ & $39.66 \pm 7.48$ & $0.007^{b, *^{*}}$ \\
\hline $20-40$ & $18(50.0)$ & $6(15.4)$ & $0.001^{\mathrm{cl} . *}$ & $18(42.9)$ & $0(0)$ & $6(33.3)$ & $0.009^{\mathrm{cl}, *}$ \\
\hline$>40$ & $18(50.0)$ & 33 (84.6) & & $24(57.1)$ & $15(100)$ & $12(66.7)$ & \\
\hline \multicolumn{8}{|l|}{ Sex } \\
\hline Male & $27(75.0)$ & 21 (53.8) & $0.057^{c)}$ & 27 (64.3) & $9(60.0)$ & $12(66.7)$ & $0.923^{c /}$ \\
\hline Female & $9(25.0)$ & 18 (46.2) & & 15 (35.7) & $6(40.0)$ & $6(33.3)$ & \\
\hline
\end{tabular}

Values are presented as number (\%).

LSTV was not found in any patient with $<20$ years of age group.

LSTV, lumbosacral transitional vertebrae; MRI, magnetic resonance imaging.

a).Independent $t$-test applied; ${ }^{b /}$ One-way analysis of variance test applied; ${ }^{\text {c) }}$ Chi-square test applied; ${ }^{*} p<0.05$.
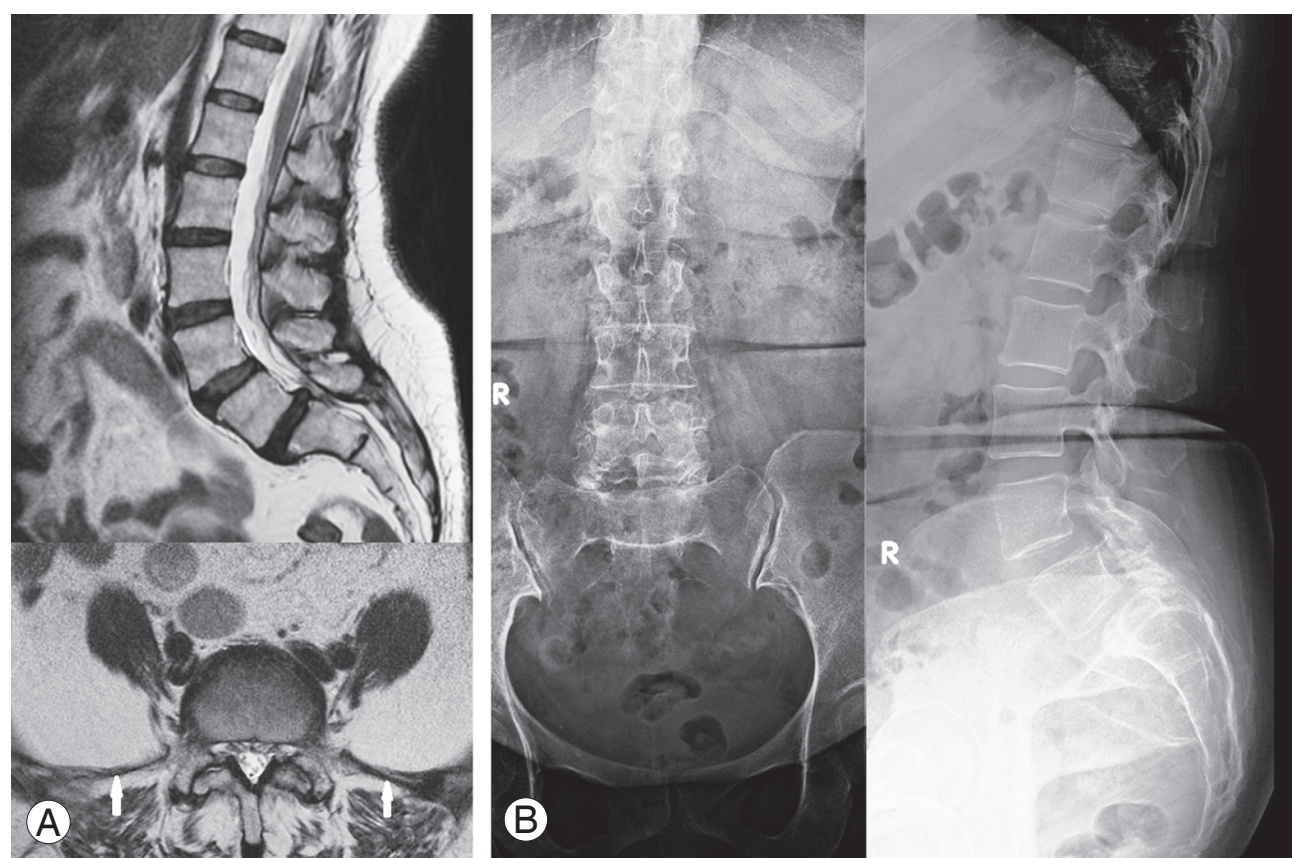

Fig. 2. Magnetic resonance imaging (MRI) (A) and plain radiographs (B) of a 61-year-old female who presented with lower back pain for 4 months. (A) MRI shows lumbosacral transition vertebra (LSTV) O'Driscoll type II. Axial section through $L 5$ shows the origin of the iliolumbar ligament from L5 (white arrows). (B) Plain radiograph shows LSTV Castellvi type II. 
observed in community-based studies [17]. The findings of the present study indicate a high prevalence of LSTV in male patients. Although this association was insignificant, a higher prevalence in male patients has also been reported in several other studies $[3,16]$.

On both T1-weighted and T2-weighted MRI, ILL is seen as a band of low signal intensity arising from the transverse process of L5 [13]. In the presence of a normal lumbosacral junction, ILL originates from L5 $[12,13,15]$. The findings of our study indicate that in the presence of a transitional lumbosacral junction, only some cases had the origin of ILL from L5 (Fig. 2). In the majority of cases, ILL did not originate from L5. Similar findings were also reported by another study [20]. Farshad-Amacker et al. [20] reported that in the setting of LSTV, the origin of ILL is quite variable, and it is not a reliable landmark for the identification of L5. It can be reliably stated that in the presence of a transitional lumbosacral junction, ILL origin does not serve as a reliable marker for the identification of L5. Therefore, sagittal and axial MRI along with coronal localizer images, if available, should be viewed together for an accurate depiction of the transitional lumbosacral junction. In addition, communication between the surgeon and radiologist is essential to avoid surgery at the wrong level.

However, our study also had a few limitations. Firstly, ILL was only assessed at L5 and in cases of non-origin from L5; other origins were not evaluated. Secondly, only MRI scans for which concomitant radiographs of the lumbar spine were available were evaluated. Patients were not exposed to any additional radiation for this study. Lastly, our study population only comprised patients with LBP. A study in an asymptomatic population can be conducted to gain further insights into this condition.

Despite these limitations, this study was the first to establish the prevalence of LSTV in a low back population from a developing country, using both plain radiographs and MRI scans. A large-scale comparative study between MRI and radiographic findings should be conducted by identifying the best MRI criteria and radiographic correlations for the improved evaluation of LSTV on MRI.

\section{Conclusions}

LSTV occurs at a high frequency in patients with LBP. Furthermore, in the presence of LSTV, ILL is not a reliable marker for the identification of L5.

\section{Conflict of Interest}

No potential conflict of interest relevant to this article was reported.

\section{References}

1. Bertolotti M. Contributo alla conoscenza dei vizi differenzazione regionale del rachide con special riguardo all assimilazione sacrale della V. Lombare. Radiol Med 1917;4:113-44.

2. Hsieh CY, Vanderford JD, Moreau SR, Prong T. Lumbosacral transitional segments: classification, prevalence, and effect on disk height. J Manipulative Physiol Ther 2000;23:483-9.

3. Nardo L, Alizai H, Virayavanich W, et al. Lumbosacral transitional vertebrae: association with low back pain. Radiology 2012;265:497-503.

4. Apazidis A, Ricart PA, Diefenbach CM, Spivak JM. The prevalence of transitional vertebrae in the lumbar spine. Spine J 2011;11:858-62.

5. Weiler C, Lopez-Ramos M, Mayer HM, et al. Histological analysis of surgical lumbar intervertebral disc tissue provides evidence for an association between disc degeneration and increased body mass index. BMC Res Notes 2011;4:497.

6. Burton AK, Balague F, Cardon G, et al. Chapter 2. European guidelines for prevention in low back pain: November 2004. Eur Spine J 2006;15 Suppl 2:S136-68.

7. Ehrlich GE. Low back pain. Bull World Health Organ 2003;81:671-6.

8. Elders LA, Burdorf A. Prevalence, incidence, and recurrence of low back pain in scaffolders during a 3-year follow-up study. Spine (Phila Pa 1976) 2004;29:E101-6.

9. Tang M, Yang XF, Yang SW, et al. Lumbosacral transitional vertebra in a population-based study of 5860 individuals: prevalence and relationship to low back pain. Eur J Radiol 2014;83:1679-82.

10. Castellvi AE, Goldstein LA, Chan DP. Lumbosacral transitional vertebrae and their relationship with lumbar extradural defects. Spine (Phila Pa 1976) 1984;9:493-5.

11. O'Driscoll CM, Irwin A, Saifuddin A. Variations in morphology of the lumbosacral junction on sagittal MRI: correlation with plain radiography. Skeletal Radiol 1996;25:225-30. 
12. Basadonna PT, Gasparini D, Rucco V. Iliolumbar ligament insertions. In vivo anatomic study. Spine (Phila Pa 1976) 1996;21:2313-6.

13. Rucco V, Basadonna PT, Gasparini D. Anatomy of the iliolumbar ligament: a review of its anatomy and a magnetic resonance study. Am J Phys Med Rehabil 1996;75:451-5.

14. Luk KD, Ho HC, Leong JC. The iliolumbar ligament. A study of its anatomy, development and clinical significance. J Bone Joint Surg Br 1986;68:197-200.

15. Pool-Goudzwaard AL, Kleinrensink GJ, Snijders CJ, Entius C, Stoeckart R. The sacroiliac part of the iliolumbar ligament. J Anat 2001;199:457-63.

16. Olofin MU, Noronha C, Okanlawon A. Incidence of lumbosacral transitional vertebrae in low back pain patients. West Afr J Radiol 2001;8:1-6.
17. Delport EG, Cucuzzella TR, Kim N, Marley J, Pruitt C, Delport AG. Lumbosacral transitional vertebrae: incidence in a consecutive patient series. Pain Physician 2006;9:53-6.

18. Masud R, Rehman K, Sadiq A, Ahmed S. Lumbosacral transitional vertebrae: incidence in patients with low back pain. Pak J Radiol 2011;21:159-62.

19. Oyinloye OI, Abdulkadir AY, Babalola OM. Incidence and patterns of lumbosacral transitional vertebrae, in patients with low backpain in a Nigerian hospital. Nig Q J Hosp Med 2009;19:95-9.

20. Farshad-Amacker NA, Lurie B, Herzog RJ, Farshad M. Is the iliolumbar ligament a reliable identifier of the L5 vertebra in lumbosacral transitional anomalies? Eur Radiol 2014;24:2623-30. 